\title{
ON A VAN DER POL TYPE EQUATION WITH DELAY IN DAMPING
}

\author{
$\mathrm{BY}$
}

\author{
GEORGE SEIFERT
}

\author{
Iowa State University, Ames, Iowa
}

We consider a delay differential equation of the form

$$
\begin{gathered}
x^{\prime \prime}+\mu\left(x^{2}(t-r)+\alpha\left(x^{\prime}(t)\right)^{2}-1\right) x^{\prime}+x=0, \quad t>0, \\
x(t)=\phi_{1}(t), \quad x^{\prime}(0)=b_{1}, \quad-r \leq t \leq 0:
\end{gathered}
$$

here $\phi_{1}(t)$ is continuous on $[-r, 0], \mu$ and $r$ are positive constants, and the prime denotes derivative with respect to the independent variable $t$.

It is well known that for $r=\alpha=0$, this equation will have nontrivial periodic solutions characterized by a so-called limit cycle in the $\left(x, x^{\prime}\right)$-plane, and that each nontrivial solution will approach such a periodic solution as $t \rightarrow \infty$. If $r>0$ and $\alpha>0$, can the same be said for solutions of (1)? The answer seems at present unknown. In the case $\alpha=r=0$, one can establish the above-mentioned result by geometric arguments in the $\left(x, x^{\prime}\right)$-plane, and use the familiar Poincaré-Bendixson theorem. These methods for the case $\alpha>0, r>0$ cannot be directly applied. In the main result of this paper we give explicit conditions on the delay $r$ in terms of $\alpha$ such that each solution $x(t)$ of $(1)$ is, together with its derivative $x^{\prime}(t)$ bounded for $t \geq 0$. Then by using a result originally due to Birkhoff, we can show the existence of nontrivial recurrent solutions. Whether all solutions are also attracted to a recurrent solution seems at present an open question.

We consider (1) as a first-order system:

$$
\begin{aligned}
& \left\{\begin{array}{l}
x^{\prime}=y, \\
y^{\prime}=\mu\left(1-x^{2}(t-r)-\alpha y^{2}(t)\right) y-x, \quad t>0 ;
\end{array}\right. \\
& x(t)=\phi_{1}(t), \quad-r \leq t \leq 0, \quad y(0)=b_{1} .
\end{aligned}
$$

Theorem 1. If $r<\min \{1, \alpha\}$, then for each solution $(x(t), y(t))$ of $(2)$ there exists a constant $B_{1}>0$ such that $x^{2}(t)+y^{2}(t)<B_{1}, t>0$.

Proof. We need only consider nontrivial solutions of (2). Define $V(x, y)=\left(x^{2}+y^{2}\right) / 2$ and $V(t)=V(x(t), y(t))$ for any such solution $(x(t), y(t))$. For $-r \leq t \leq 0$, we take $y(t)=b_{1}$. So $V(t)$ is defined for $t \geq-r$, and all $t$ for which $(x(t), y(t))$ exists. Using (2) 
we find that

$$
V^{\prime}(t)=\mu\left(1-x^{2}(t-r)-\alpha y^{2}(t)\right) y^{2}(t), \quad t>0 .
$$

Let $B_{0}>0$ be so large that $V(x, y)>B_{0}$ implies

$$
(1-r) x^{2}+(\alpha-r) y^{2}>2 .
$$

Let $B_{1}>B_{0}$ satisfy $\phi_{1}^{2}(t)+b_{1}^{2}<B_{1},-r \leq t \leq 0$, and suppose for some $t_{1}>0$, $V\left(t_{1}\right)>B_{1}$. Then there exists a $t_{0}>0$ such that $V\left(t_{0}\right)=B_{1}, V(t)<B_{1}, 0 \leq t<t_{0}$. Using the Mean Value Theorem, there exists a sequence $t_{n}<t_{0}, n=1,2, \ldots$, such that $t_{n} \rightarrow t_{0}$ and $V^{\prime}\left(t_{n}\right)>0$. Let $\varepsilon>0$ be such that $2 r \varepsilon<1$. Since $V(t)<V\left(t_{0}\right)$, $-r \leq t<t_{0}$, it follows that

$$
V(s) \leq V\left(t_{n}\right)+\varepsilon, \quad t_{n}-r \leq s \leq t_{n}
$$

for $n$ sufficiently large. Using the fact that $d\left(x^{2}(t)\right) / d t=2 x(t) y(t)$, we get $x^{2}(t)-$ $x^{2}(t-r)=2 \int_{t-r}^{t} x(s) y(s) d s$ where again we take $y(s)=b_{1}$, for $-r \leq s \leq 0$. Using this in (3), we get

$$
\begin{aligned}
V^{\prime}(t) & =\mu\left(1-x^{2}(t)-\alpha y^{2}(t)+2 \int_{t-r}^{t} x(s) y(s) d s\right) y^{2}(t) \\
& \leq \mu\left(1-x^{2}(t)-\alpha y^{2}(t)+2 \int_{t-r}^{t} V(s) d s\right) y^{2}(t)
\end{aligned}
$$

and using this with $t=t_{n}, n$ sufficiently large, and (5) we get

$$
\begin{aligned}
V^{\prime}\left(t_{n}\right) & \leq \mu\left(1-x^{2}\left(t_{n}\right)-2 y^{2}\left(t_{n}\right)+2 V\left(t_{n}\right)+2 r \varepsilon\right) y^{2}\left(t_{n}\right) \\
& =\mu\left(1-(1-r) x^{2}\left(t_{n}\right)-(\alpha-r) y^{2}\left(t_{n}\right)+2 r \varepsilon\right) y^{2}\left(t_{n}\right) .
\end{aligned}
$$

Since $\left(x\left(t_{n}\right), y\left(t_{n}\right)\right) \rightarrow\left(x\left(t_{0}\right), y\left(t_{0}\right)\right)$ as $n \rightarrow \infty$, and $V\left(x\left(t_{0}\right), y\left(t_{0}\right)\right)>B_{0}$, we get, using (4), $V^{\prime}\left(t_{n}\right) \leq \mu(1-2+2 r \varepsilon) y^{2}\left(t_{n}\right) \leq 0$ for $n$ sufficiently large, which contradicts $V^{\prime}\left(t_{n}\right)>$ 0 ; this proves our theorem.

We remark that under the conditions of Theorem 1, each solution of (2) exists for all $t>0$; cf. Theorem 3.2 in [1].

To introduce the concept of recurrent solution of (2), we need some notation and definitions.

For any $t \geq 0$ and function $x(t)$ continuous for $t \geq-r$, we use the notation $x_{t}=$ $x(t+s),-r \leq s \leq 0$, and if $x(t)=\phi(t),-r \leq t \leq 0$, we put $x_{t}=x_{t}(\phi)$.

Let $C=\{(\phi(s), b),-r \leq s \leq 0: \phi$ is continuous on $[-r, 0], b$ is real $\}$.

Note that for a solution $(x(t), y(t))$ of $(2),\left(x_{t}\left(\phi_{1}\right), y(t)\right) \in C, t \geq 0$.

If $(\phi, b)$ and $(\psi, c)$ are in $C$ we define

$$
d((\phi, b),(\psi, c))=\sup \left\{\left[(\phi(s)-\psi(s))^{2}+(b-c)^{2}\right]^{1 / 2}, s \in[-r, 0]\right\} .
$$

Then $\{C, d\}$ is a metric space with metric $d(\cdot, \cdot)$.

In the following definitions, we denote by $\phi$ the elements (points) of $C$.

Definition 1. A semiflow on $C$ is a function $\Phi(t, \phi)$ continuous on $[0, \infty] \times C$ to $C$ such that $\Phi(0, \phi)=\phi$ and

$$
\Phi\left(t_{1}+t_{2}, \phi\right)=\Phi\left(t_{1}, \Phi\left(t_{2}, \phi\right)\right) \quad \text { for } t_{1} \geq 0, t_{2} \geq 0, \phi \in C .
$$


Definition 2. A point $\phi \in C$ is called a recurrent point for the semiflow $\Phi$ if given $\varepsilon>0$, there exists an $L(\varepsilon)>0$ such that given any $t \geq 0, s \geq 0$, there exists a $\tau, t \leq \tau \leq t+L(\varepsilon)$ such that

$$
d(\Phi(\tau, \phi), \Phi(s, \phi))<\varepsilon
$$

Note that if for some $T>0, \Phi(t+T, \phi)=\Phi(t, \phi), t \geq 0$, then $\phi$ is a recurrent point; we need only take $L(\varepsilon)=T$, and $\tau=s+n T$ for a suitable integer $n$. In this case $\phi$ is said to be a periodic point.

This definition of recurrence is essentially that given in [2], 7.05, and is what is called uniform recurrence in [3].

Definition 3. For $\phi \in C$, define

$$
\begin{aligned}
\omega(\phi)=\left\{\psi \in C: \text { there exists a sequence } t_{n}>0, t_{n}\right. & \rightarrow \infty \text { as } n \rightarrow \infty, \\
& \text { and } \left.\Phi\left(t_{n}, \phi\right) \rightarrow \psi \text { as } n \rightarrow \infty\right\} .
\end{aligned}
$$

Definition 4. $0^{+}(\phi)=\{\Phi(t, \phi): t \geq 0\}$.

We note that the above definitions can be made if $\{C, d\}$ is any metric space, not just the special case $\{C, d\}$ given above. For a good discussion of semiflows on more general spaces, cf. the book by Saperstone [3].

The following result is well known for general semiflows on a metric space, cf. [2], pp. 338-344.

Lemma 1. If the set $0^{+}(\phi)$ has compact closure $\overline{0^{+}(\phi)}$, then $\omega(\phi)$ is nonempty, compact, and connected, and clearly $\omega(\phi) \subset \overline{0^{+}(x)}$.

It is a consequence of a result originally due to G. D. Birkhoff that if $\overline{0^{+}(\phi)}$ is compact, then $\omega(\phi)$ contains recurrent points; cf. [2], 7.02 or Theorem 3.9 in [3].

Since (2) is an autonomous delay differential equation whose solutions are uniquely determined by $\left(\phi_{1}(t), b_{1}\right),-r \leq t \leq 0$ and exist for $t>0$, these solutions generate a semiflow on $C$ defined by $\left(x_{t}\left(\phi_{1}\right), y(t)\right), t \geq 0, y(0)=b_{1}$.

Definition 5. The solution $(x(t), y(t))$ is called recurrent if the point $\left(\phi_{1}, b_{1}\right) \in C$ is a recurrent point for the semiflow generated by solutions of (2) as described above.

We note that if there exists a $T>0$ such that the solution $(x(t), y(t))$ of $(2)$ satisfies $(x(t+T), y(t+T))=(x(t), y(t)), t \geq 0$, then this solution is clearly recurrent. It does not however seem to follow that recurrent solutions of (2) have this periodicity property. In fact, it is known that there exist semiflows with recurrent points that are not periodic and are known as almost periodic points; cf. [2], 8.10.

Lemma 2. If $(x(t), y(t))$ is a solution of (2), then in terms of the semiflow on $C$ generated by solutions of $(2), 0^{+}(\phi)$ has compact closure; here $\phi=\left(\phi_{1}, b_{1}\right) \in C$, and as in Theorem $1, r<\min \{1, \alpha\}$.

A proof of this lemma can be based on the completely continuous property of the so-called solution map for (2); cf. [1], 3.6, using the fact that each solution of (2) is bounded on $(0, \infty)$.

We can now state the second main result of this paper. 
THEOREM 2. If $r<\min \{1, \alpha\}$, (2) will have nontrivial recurrent solutions, i.e., recurrent solutions distinct from $(x(t), y(t))=(0,0)$ for all $t>0$.

Proof. Let $(x(t), y(t))$ be a solution of (2) with $\phi_{1}^{2}(t)+b_{1}^{2}>0,-r \leq t \leq 0$. The fact that the trivial solution $(0,0)$ of $(2)$ is completely unstable in the sense that there exists a $\delta>0$ such that $0<\phi_{1}^{2}(t)+b_{1}^{2}<\delta^{2},-r \leq t \leq 0$, implies $x^{2}(t)+y^{2}(t)>\delta^{2}$ for $t \geq t_{1}$ for some $t_{1}>0$ shows clearly that $\overline{0^{+}(\phi)}$ cannot contain the trivial point $\left(\phi_{0}, 0\right)$, where $\phi_{0}=\phi(s)=0,-r \leq s \leq 0$, and so $\omega(\phi), \phi=\left(\phi_{1}, b_{1}\right)$, also cannot contain this trivial point. The complete instability of the trivial solution of (2) follows easily from the fact that the linearization of (2) with respect to the trivial solution is

$$
\left\{\begin{array}{l}
x^{\prime}=y \\
y^{\prime}=\mu y-x
\end{array}\right.
$$

whose characteristic roots have positive real parts. Thus our theorem follows since, for $\psi \in \omega(\phi)$ and recurrent, the solution $(x(t), y(t))$ with $x(t)=\psi_{1}(t),-r \leq t \leq 0, y(0)=b_{2}$ is recurrent; here $\psi=\left(\psi_{1}(s), b_{2}\right),-r \leq s \leq 0$.

Some concluding remarks are in order. First, using similar methods we can easily obtain results like Theorems 1 and 2 for an equation of the form:

$$
x^{\prime \prime}+\mu\left(x^{2}(t-r)+\alpha\left(x^{\prime}(t-r)\right)^{2}-1\right) x^{\prime}(t)+x=0 ;
$$

only obvious modifications are needed; i.e., in place of the space $C$ we may now use the space $\left\{\phi_{1}(s), \phi_{2}(s):-r \leq s \leq 0, \phi_{i}(s), i=1,2\right.$ is continuous $\}$.

We also can deal with much more general equations like

$$
x^{\prime \prime}+f\left(x(t), x^{\prime}(t), x(t-r), x^{\prime}(t-r)\right) x^{\prime}+g(x)=0
$$

and under suitable but more complicated conditions on $f$ and $g$ and get results like our Theorems 1 and 2, using the methods similar to those used in the simpler special case (1).

Next, we note that delay differential equations of the form

$$
x^{\prime \prime}+f(x) x^{\prime}+g(x(t-r))=e(t)
$$

have been studied for some time. A recent paper by Burton and Zhang [4] gives conditions under which the solutions of this equation have certain boundedness and stability properties, and if $e(t)$ is of period $T$, that there exist solutions of period $T$. If $e(t)=0$ for all $t$ and $f(x)$ and $x g(x)$ are positive for all $x \neq 0$ there are many papers giving conditions for the asymptotic stability of the trivial solution; cf. the references cited in [4].

Finally we note that if $r>0$ and $\alpha=0$, the methods used in the proof of Theorem 1 fail. An open question seems to be whether in this case, solutions of (2) are bounded on $(0, \infty)$ for $r$ sufficiently small. More general results for delay differential equations with sufficiently small delays may be relevant; cf. [5], p. 81. However, this result seems to show only that for such delay equations the solutions are also solutions of an ordinary differential equation. Whether in our specific case, this result can show that all solutions of (2) are bounded for $t \geq 0$ is not immediately obvious unless one can get explicit information about the associated ordinary differential equation. 
The author is indebted to the referee for pointing out this result in [5].

\section{REFERENCES}

[1] J. K. Hale, Theory of Functional Differential Equations, Appl. Math. Sci. 3, Springer-Verlag, New York, 1977.

[2] V. V. Nemytskii and V. V. Stepanov, Qualitative Theory of Differential Equations, Princeton Univ. Press, 1960.

[3] S. H. Saperstone, Semidynamical Systems in Infinite Dimensional Spaces, Appl. Math. Sci. 37, Springer-Verlag, New York, 1981.

[4] T. A. Burton and Bo Zhang, Boundedness, periodicity and convergence of solutions in a retarded Liénard equation, Ann. Mat. Pura Appl. (IV) CLXV, 351-368 (1993).

[5] J. K. Hale, L. T. Magalhães, and W. M. Oliva, An Introduction to Infinite Dimensional SystemsGeometric Theory, Applied Math. Sci. 47, Springer-Verlag, New York, Berlin, 1984. 